\title{
Completely positive maps for imprimitive complex reflection groups
}

\section{Randriamaro $\mathrm{H}$.}

In 1994, M. Bożejko and R. Speicher proved the existence of completely positive quasimultiplicative maps from the group algebra of Coxeter groups to the set of bounded operators. They used some of them to define an inner product associated to creation and annihilation operators on a direct sum of Hilbert space tensor powers called full Fock space. Afterwards, A. Mathas and R. Orellana defined in 2008 a length function on imprimitive complex reflection groups that allowed them to introduce an analogue to the descent algebra of Coxeter groups. In this article, we use the length function defined by A. Mathas and R. Orellana to extend the result of M. Bożejko and R. Speicher to imprimitive complex reflection groups, in other words to prove the existence of completely positive quasimultiplicative maps from the group algebra of imprimitive complex reflection groups to the set of bounded operators. Some of those maps are then used to define a more general inner product associated to creation and annihilation operators on the full Fock space. Recall that in quantum mechanics, the state of a physical system is represented by a vector in a Hilbert space, and the creation and annihilation operators act on a Fock state by respectively adding and removing a particle in the ascribed quantum state.

Key words and phrases: bounded operator, quasimultiplicative map, Fock space.

Lot II B 32 bis Faravohitra, 101 Antananarivo, Madagascar

E-mail: hery.randriamaro@gmail.com

\section{Introduction}

Denote by $\mathbb{N}$ the set of positive integers as usual, by $[i, j]$ the set $\{i, i+1, \ldots, j\}$ for integers $i, j$ with $i \leq j$, and by $[j]$ the set $[1, j]$. Recall that, for $m, n \in \mathbb{N}$, the complex reflection group $G(m, 1, n)$ is generated by reflections $s_{0}, s_{1}, s_{2}, \ldots, s_{n-1}$ on $\mathbb{C}^{n}$ subject to the relations

$$
\begin{aligned}
& s_{0}^{m}=s_{1}^{2}=s_{2}^{2}=\cdots=s_{n-1}^{2}=1 \text {, } \\
& s_{0} s_{1} s_{0} s_{1}=s_{1} s_{0} s_{1} s_{0}, \\
& \forall i \in[n-2]: s_{i} s_{i+1} s_{i}=s_{i+1} s_{i} s_{i+1} \text {, } \\
& \forall i, j \in[0, n-1],|i-j| \geq 2: s_{i} s_{j}=s_{j} s_{i} \text {. }
\end{aligned}
$$

The study of cyclotomic descent algebras by A. Mathas and R. Orellana [7, § 2] leads to consider the reflections $t_{1}:=s_{0}$, and $t_{i+1}:=s_{i} t_{i} s_{i}$ for $i \in[n-1]$. The subgroup $T:=\left\langle t_{1}, t_{2}, \ldots, t_{n}\right\rangle$ is isomorphic to $(\mathbb{Z} / m \mathbb{Z})^{n}$, and is normal in $G(m, 1, n)$. Since $\left\langle s_{1}, s_{2}, \ldots, s_{n-1}\right\rangle$ is the symmetric group $\operatorname{Sym}(n)$ of order $n$, then $G(m, 1, n)=T \rtimes \operatorname{Sym}(n)$. As set, we have

$$
G(m, 1, n)=\left\{t_{1}^{\alpha_{1}} t_{2}^{\alpha_{2}} \ldots t_{n}^{\alpha_{n}} w \mid \alpha_{1}, \alpha_{2}, \ldots, \alpha_{n} \in[0, m-1], w \in \operatorname{Sym}(n)\right\} .
$$

$\mathrm{Y} \Delta \mathrm{K} 512.54$

2020 Mathematics Subject Classification: 05E15, 47L30, 81R10.

This research was funded by the mother of the author. 
Hence, the set $\Pi=\left\{t_{1}, t_{2}, \ldots, t_{n}, s_{1}, s_{2}, \ldots, s_{n-1}\right\}$ generates $G(m, 1, n)$ so that A. Mathas and R. Orellana could define the length function $1: G(m, 1, n) \rightarrow \mathbb{N}$ given by [7, Definition 2.3]

$$
1(g):=\min \left\{k \in \mathbb{N} \mid \exists r_{1}, r_{2}, \ldots, r_{k} \in \Pi: g=r_{1} r_{2} \ldots r_{k}\right\}
$$

Let call $\Pi$ a set of representative reflections of $G(m, 1, n)$. In addition to those above, the reflections in $\Pi$ are also subject to the relations

$$
\forall i, j \in[n], i \neq n,|i-j| \geq 2: s_{i} t_{i} s_{i} t_{i}=t_{i} s_{i} t_{i} s_{i}, s_{i} t_{i+1} s_{i} t_{i+1}=t_{i+1} s_{i} t_{i+1} s_{i}, s_{i} t_{j}=t_{j} s_{i}
$$

with following Dynkin diagram.

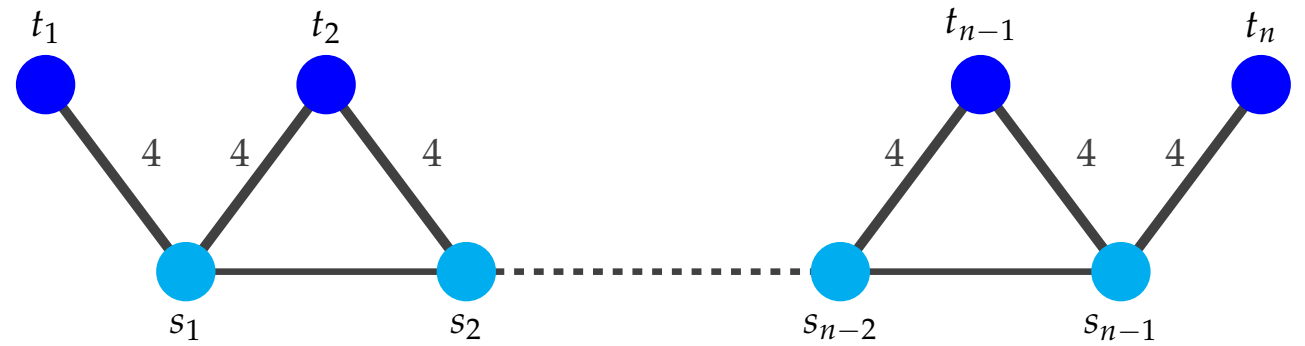

We work on a complex Hilbert space $\mathbf{H}$ endowed with an inner product $\langle., \cdot\rangle: \mathbf{H} \times \mathbf{H} \rightarrow \mathbb{C}$. Denoting by $\mathbb{R}_{+}$the set of nonnegative real numbers, recall that $\mathbf{H}$ is also a normed space with norm $\|\cdot\|: \mathbf{H} \rightarrow \mathbb{R}_{+}$defined by $\|x\|:=\sqrt{\langle x, x\rangle}$.

A bounded operator on $\mathbf{H}$ is a linear operator $\mathrm{L}: \mathbf{H} \rightarrow \mathbf{H}$ for which there exists a number $\alpha \in \mathbb{R}_{+}$such that, for every $x \in \mathbf{H},\|L x\| \leq \alpha\|x\|$. The set $\mathscr{B}_{\mathbf{H}}$ of bounded operators on $\mathbf{H}$ is a normed algebra with norm $\|\cdot\|_{\text {op }}$ defined, for every bounded operator $L$, by

$$
\|\mathrm{L}\|_{\mathrm{op}}:=\inf \left\{\alpha \in \mathbb{R}_{+} \mid \forall x \in \mathbf{H}:\|\mathrm{L} x\| \leq \alpha\|x\|\right\} \text {. }
$$

The adjoint of $\mathrm{L} \in \mathscr{B}_{\mathbf{H}}$ is the linear operator $\mathrm{L}^{*}: \mathbf{H} \rightarrow \mathbf{H}$ such that $\left\langle\mathrm{L}^{*} x, y\right\rangle=\langle x, \mathrm{~L} y\rangle$ for every $x, y \in \mathbf{H}$. Recall that $\mathrm{L}$ is said self-adjoint if $\mathrm{L}^{*}=\mathrm{L}$.

Let $f=\sum_{u \in G(m, 1, n)} f(u) u$ and $g=\sum_{u \in G(m, 1, n)} g(u) u$ belong to $\mathbb{C} G(m, 1, n)$. The group algebra $\mathbb{C} G(m, 1, n)$ is a Hilbert space for the inner product $\langle., \cdot\rangle: \mathbb{C} G(m, 1, n) \times \mathbb{C} G(m, 1, n) \rightarrow \mathbb{C}$ defined by $\langle f, g\rangle:=\sum_{u \in G(m, 1, n)} \overline{f(u)} g(u)$. Moreover, as the map $g \mapsto f g$ is a linear operator on $\mathbb{C} G(m, 1, n)$, we may define the adjoint of $f$ by $f^{*}:=\sum_{u \in G(m, 1, n)} \overline{f\left(u^{-1}\right)} u \in \mathbb{C} G(m, 1, n)$.

A linear map $\varphi: \mathbb{C} G(m, 1, n) \rightarrow \mathscr{B}_{\mathbf{H}}$ is quasimultiplicative if $\varphi(1)=\mathrm{id}_{\mathbf{H}}$ and

$$
\forall u, v \in G(m, 1, n): 1(u v)=1(u)+1(v) \Rightarrow \varphi(u v)=\varphi(u) \varphi(v) .
$$

A linear map $\varphi: \mathbb{C} G(m, 1, n) \rightarrow \mathscr{B}_{\mathbf{H}}$ is completely positive if

$$
\forall k \in \mathbb{N}^{*}, f_{1}, \ldots, f_{k} \in \mathbb{C} G(m, 1, n), x_{1}, \ldots, x_{k} \in \mathbf{H}:\left\langle\sum_{i, j=1}^{k} \varphi\left(f_{j}^{*} f_{i}\right) x_{i}, x_{j}\right\rangle \geq 0 .
$$


Theorem 1. Let $\Pi=\left\{t_{1}, t_{2}, \ldots, t_{n}, s_{1}, s_{2}, \ldots, s_{n-1}\right\}$ be a representative reflection set of the complex reflection group $G(m, 1, n)$, and $\mathrm{S}_{1}, \ldots, \mathrm{S}_{n-1}, \mathrm{~T}_{1}, \ldots, \mathrm{T}_{n} \in \mathscr{B}_{\mathbf{H}}$ such that

- $\forall i \in[n-1]:\left\|S_{i}\right\|_{\text {op }} \leq 1, S_{i}^{*}=S_{i}$

- $\forall j \in[n]:\left\|\mathrm{T}_{j}\right\|_{\text {op }} \leq 1, \sum_{i \in[m-1]}\left(\mathrm{T}_{j}^{i}\right)^{*}=\sum_{i \in[m-1]} \mathrm{T}_{j}^{i}$

- the bounded operators satisfy the following braid relations:

$$
\begin{aligned}
& \forall i \in[n-2]: \mathrm{S}_{i} \mathrm{~S}_{i+1} \mathrm{~S}_{i}=\mathrm{S}_{i+1} \mathrm{~S}_{i} \mathrm{~S}_{i+1}, \quad \forall i, j \in[n-1],|i-j| \geq 2: \mathrm{S}_{i} \mathrm{~S}_{j}=\mathrm{S}_{j} \mathrm{~S}_{i}, \\
& \forall i, j \in[n]: \mathrm{T}_{i} \mathrm{~T}_{j}=\mathrm{T}_{j} \mathrm{~T}_{i}, \quad \forall i, j \in[n], i \neq n,|i-j| \geq 2: \mathrm{S}_{i} \mathrm{~T}_{j}=\mathrm{T}_{j} \mathrm{~S}_{i}, \\
& \forall i \in[n-1]: \mathrm{S}_{i} \mathrm{~T}_{i} \mathrm{~S}_{i} \mathrm{~T}_{i}=\mathrm{T}_{i} \mathrm{~S}_{i} \mathrm{~T}_{i} \mathrm{~S}_{i}, \mathrm{~S}_{i} \mathrm{~T}_{i+1} \mathrm{~S}_{i} \mathrm{~T}_{i+1}=\mathrm{T}_{i+1} \mathrm{~S}_{i} \mathrm{~T}_{i+1} \mathrm{~S}_{i} .
\end{aligned}
$$

Then, the quasimultiplicative linear $\operatorname{map} \varphi: \mathbb{C} G(m, 1, n) \rightarrow \mathscr{B}_{\mathbf{H}}$ given by

$$
\varphi(1)=\mathrm{id}_{\mathbf{H}}, \quad \forall i \in[n-1]: \varphi\left(s_{i}\right)=\mathrm{S}_{i} \quad \text { and } \quad \forall j \in[n]: \varphi\left(t_{j}\right)=\mathrm{T}_{j}
$$

is completely positive.

Remark that we recover the completely positive maps on symmetric groups [2, Theorem 1.1] by letting $\mathrm{T}_{j}=0$, and those on commutative groups [2, Theorem 5.1] by letting $\mathrm{S}_{i}=0$ in that theorem. If in addition we assume that $S_{i}^{2}=\mathrm{id}_{\mathbf{H}}$ and $\mathrm{T}_{j}^{m}=\mathrm{id}_{\mathbf{H}}$ in Theorem 1 , then $\varphi$ would be a group algebra representation of $\mathbb{C} G(m, 1, n)$.

In the following, we describe important bounded operators of $\mathscr{B}_{\mathbf{H}^{\otimes n}}$ fulfilling the assumptions of Theorem 1 . Take some vector $\Omega \in \mathbf{H}$ with $\|\Omega\|=1$ called vacuum. Denoting by $\mathbb{Z}_{+}$ the set of nonnegative integers, the full Fock space for $\mathbf{H}$ is $\bigoplus_{n \in \mathbb{Z}_{+}} \mathbf{H}^{\otimes n}$ where $\mathbf{H}^{0}:=\mathbb{C} \Omega$.

Assume that we are given some operators $S, T$ such that

- $\mathrm{S} \in \mathscr{B}_{\mathbf{H} \otimes \mathbf{H}},\|\mathrm{S}\|_{\text {op }}<1, \mathrm{~S}^{*}=\mathrm{S}$, and $\mathrm{S}$ fulfills the braid relation also called Yang-Baxter equation $\left(\mathrm{id}_{\mathbf{H}} \otimes \mathrm{S}\right)\left(\mathrm{S} \otimes \mathrm{id}_{\mathbf{H}}\right)\left(\mathrm{id}_{\mathbf{H}} \otimes \mathrm{S}\right)=\left(\mathrm{S} \otimes \mathrm{id}_{\mathbf{H}}\right)\left(\mathrm{id}_{\mathbf{H}} \otimes \mathrm{S}\right)\left(\mathrm{S} \otimes \mathrm{id}_{\mathbf{H}}\right)[6, \S 8.1]$,

- $\mathrm{T} \in \mathscr{B}_{\mathbf{H}},\|\mathrm{T}\|_{\text {op }}<1, \sum_{i \in[m-1]}\left(\mathrm{T}^{i}\right)^{*}=\sum_{i \in[m-1]} \mathrm{T}^{i}$, and $\mathrm{T}$ fulfills the relations

$\left(T \otimes \mathrm{id}_{\mathbf{H}}\right) \mathrm{S}\left(\mathrm{T} \otimes \mathrm{id}_{\mathbf{H}}\right) \mathrm{S}=\mathrm{S}\left(\mathrm{T} \otimes \mathrm{id}_{\mathbf{H}}\right) \mathrm{S}\left(\mathrm{T} \otimes \mathrm{id}_{\mathbf{H}}\right)$,

$\left(\mathrm{id}_{\mathbf{H}} \otimes \mathrm{T}\right) \mathrm{S}\left(\mathrm{id}_{\mathbf{H}} \otimes \mathrm{T}\right) \mathrm{S}=\mathrm{S}\left(\mathrm{id}_{\mathbf{H}} \otimes \mathrm{T}\right) \mathrm{S}\left(\mathrm{id}_{\mathbf{H}} \otimes \mathrm{T}\right)$.

Let $n \in \mathbb{N}, i \in[n-1]$, and $j \in[n]$. Define the operators $\mathrm{S}_{i}, \mathrm{~T}_{j} \in \mathscr{B}_{\mathbf{H}^{\otimes n}}$ by

$\mathrm{S}_{i}:=\overbrace{\mathrm{id}_{\mathbf{H}} \otimes \cdots \otimes \mathrm{id}_{\mathbf{H}}}^{i-1 \text { times }} \otimes \mathbf{S} \otimes \overbrace{\mathrm{id}_{\mathbf{H}} \otimes \cdots \otimes \mathrm{id}_{\mathbf{H}}}^{n-i-1 \text { times }}$ and $\mathrm{T}_{j}:=\overbrace{\mathrm{id}_{\mathbf{H}} \otimes \cdots \otimes \mathrm{id}_{\mathbf{H}}}^{j-1 \text { times }} \otimes \mathbf{T} \otimes \overbrace{\mathrm{id}_{\mathbf{H}} \otimes \cdots \otimes \mathrm{id}_{\mathbf{H}}}^{n-j \text { times }}$.

The $\mathrm{S}_{i}{ }^{\prime} \mathrm{s}$ and $\mathrm{T}_{j}$ 's fulfill the assumptions of Theorem 1. Define $\mathrm{P}_{n}: \mathbf{H}^{\otimes n} \rightarrow \mathbf{H}^{\otimes n}$ as the operator given by

$$
\mathrm{P}_{0}:=\operatorname{id}_{\mathbb{C} \Omega} \text { and } \mathrm{P}_{n}:=\sum_{u \in G(m, 1, n)} \varphi(u),
$$

where $\varphi$ is the quasimultiplicative map of Theorem 1 with $\varphi\left(s_{i}\right)=\mathrm{S}_{i}$ and $\varphi\left(t_{j}\right)=\mathrm{T}_{j}$. 
Theorem 2. The sesquilinear form $\langle\cdot, \cdot\rangle_{G}: \bigoplus_{n \in \mathbb{Z}_{+}} \mathbf{H}^{\otimes n} \times \bigoplus_{n \in \mathbb{Z}_{+}} \mathbf{H}^{\otimes n} \rightarrow \mathbb{C}$ given by

$$
\forall X \in \mathbf{H}^{\otimes k}, \forall Y \in \mathbf{H}^{\otimes n}:\langle X, Y\rangle_{G}:=\delta_{k n}\left\langle X, \mathrm{P}_{n} Y\right\rangle
$$

is an inner product so that, for each $x \in \mathbf{H}$, one can define creation and annihilation operators $\mathrm{d}^{*}(x): \bigoplus_{n \in \mathbb{Z}_{+}} \mathbf{H}^{\otimes n} \rightarrow \bigoplus_{n \in \mathbb{Z}_{+}} \mathbf{H}^{\otimes n}$ and $\mathrm{d}(x): \bigoplus_{n \in \mathbb{Z}_{+}} \mathbf{H}^{\otimes n} \rightarrow \bigoplus_{n \in \mathbb{Z}_{+}} \mathbf{H}^{\otimes n}$ respectively, which are adjoint with respect to $\langle\cdot, \cdot\rangle_{G}$. Moreover, we have $\left\|\mathrm{d}^{*}(x)\right\|_{\mathrm{op}} \leq \frac{\|x\|}{\sqrt{\left(1-\|\mathrm{S}\|_{\mathrm{op}}\right)\left(1-\|\mathrm{T}\|_{\mathrm{op}}\right)}}$.

The inner product defined by M. Bożejko and R. Speicher [2, Theorem 3.1] is naturally a special case of $\langle\cdot, \cdot \cdot\rangle_{G}$. Note that, letting $\left\{e_{i}\right\}_{i \in I}$ be some basis of $\mathbf{H}$ and $q_{i j} \in \mathbb{C}$, they used their inner product associated to creation and annihilation operators $\mathrm{d}^{*}\left(e_{j}\right)$ and $\mathrm{d}\left(e_{i}\right)$ to construct the Fock representation of the $q_{i j}$-relations [2, Corollary 3.2]

$$
\mathrm{d}\left(e_{i}\right) \mathrm{d}^{*}\left(e_{j}\right)-q_{i j} \mathrm{~d}^{*}\left(e_{j}\right) \mathrm{d}\left(e_{i}\right)=\delta_{i j},
$$

where $q_{j i}=\bar{q}_{i j}$ and $\left|q_{i j}\right| \leq 1$. In [8], S. Meljanac and D. Svrtan independently did the same construction. That $q_{i j}$-relation model is a generalization of previously studied models $[5,10,12]$. But we recently established a more general $q_{i j}$-relation model for arbitrary $q_{i j}$ (see [9]). Furthermore, other creation and annihilation operators have been established over time like those of V. Bargmann [1] and J. Stochel [11] defined from the complex holomorphic functions on $\mathbb{C}^{n}$. And newly, A. Daletskii, A. Kalyuzhny, E. Lytvynov, and D. Proskurin defined such operators for which the associated Hilbert space is the $L^{2}$-space of $\mathbb{C}^{m}$-valued functions on $\mathbb{R}^{n}$ [3].

We use the proof strategy of M. Bożejko and R. Speicher to prove in Section 2 the complete positivity of the quasimultiplicative linear map $\varphi$ in Theorem 1, and to build in Section 3 the inner product associated with creation and annihilation operators in Theorem 2.

\section{Completely positive maps}

We prove the complete positivity of the map $\varphi$ in Theorem 1 in this section.

Lemma 1. Let $\Pi=\left\{t_{1}, t_{2}, \ldots, t_{n}, s_{1}, s_{2}, \ldots, s_{n-1}\right\}$ be a representative reflection set of the complex reflection group $G(m, 1, n)$, and $\varphi$ the quasimultiplicative map of Theorem 1 . If $\|\varphi(r)\|_{\text {op }}<1$ for every $r \in \Pi$, then the operator $\mathrm{P}:=\sum_{u \in G(m, 1, n)} \varphi(u) \in \mathscr{B}_{\mathbf{H}}$ is invertible.

Proof. We have $\mathrm{P}=\sum_{t \in T} \varphi(t) \sum_{w \in \operatorname{Sym}(n)} \varphi(w)$. On one side, M. Bożejko and R. Speicher already proved that $\sum_{w \in \operatorname{Sym}(n)} \varphi(w)$ is invertible [2, Theorem 2.4]. On the other side,

$$
\sum_{t \in T} \varphi(t)=\prod_{j \in[n]} \sum_{i \in[0, m-1]} \varphi\left(t_{j}\right)^{i}
$$

Remark that $\left(\mathrm{id}_{\mathbf{H}}-\varphi\left(t_{j}\right)\right) \sum_{i \in[0, m-1]} \varphi\left(t_{j}\right)^{i}=\mathrm{id}_{\mathbf{H}}-\varphi\left(t_{j}\right)^{m}$. Since $\left\|\varphi\left(t_{j}\right)^{m}\right\|_{\text {op }}<\left\|\varphi\left(t_{j}\right)\right\|_{\text {op }}<1$, both operators $\mathrm{id}_{\mathbf{H}}-\varphi\left(t_{j}\right)^{m}$ and $\mathrm{id}_{\mathbf{H}}-\varphi\left(t_{j}\right)$ are consequently invertible. Hence the operator $\sum_{i \in[0, m-1]} \varphi\left(t_{j}\right)^{i}$ is invertible with inverse $\left(\mathrm{id}_{\mathbf{H}}-\varphi\left(t_{j}\right)\right) \sum_{k \in \mathbb{N}} \varphi\left(t_{j}\right)^{m k}$. 
An operator $L \in \mathscr{B}_{\mathbf{H}}$ is said to be positive and strictly positive if, respectively, $\langle\mathrm{L} x, x\rangle \geq 0$ and $\langle\mathrm{L} x, x\rangle>0$ for every nonzero vector $x \in \mathbf{H}$.

Lemma 2. Let $\Pi=\left\{t_{1}, t_{2}, \ldots, t_{n}, s_{1}, s_{2}, \ldots, s_{n-1}\right\}$ be a representative reflection set of the complex reflection group $G(m, 1, n)$, and $\varphi$ the quasimultiplicative map of Theorem 1 . If $\|\varphi(r)\|_{\text {op }}<1$ for every $r \in \Pi$, then the operator $\mathrm{P}=\sum_{u \in G(m, 1, n)} \varphi(u)$ is strictly positive.

Proof. M. Bożejko and R. Speicher defined for a self-adjoint operator $L \in \mathscr{B}_{\mathbf{H}}$ the number

$$
\mathrm{m}_{0}(\mathrm{~L}):=\inf \{\langle\mathrm{L} x, x\rangle \in \mathbb{R} \mid x \in \mathbf{H},\|x\|=1\},
$$

which is in fact the smallest element in the spectrum of $L$, and proved that, for $L, K \in \mathscr{B}_{\mathbf{H}}$ self-adjoint, $\left|\mathrm{m}_{0}(\mathrm{~L})-\mathrm{m}_{0}(\mathrm{~K})\right| \leq\|\mathrm{L}-\mathrm{K}\|_{\text {op }}$ [2, Lemma 2.5].

If $0 \leq q \leq 1$, let $\varphi_{q}: \mathbb{C} G(m, 1, n) \rightarrow \mathscr{B}_{\mathbf{H}}$ be the quasimultiplicative linear map such that, for every $r \in \Pi, \varphi_{q}(r):=q \varphi(r)$. The operator $\mathrm{P}_{q}:=\sum_{u \in G(m, 1, n)} \varphi_{q}(u) \in \mathscr{B}_{\mathbf{H}}$ is self-adjoint since

$$
\begin{aligned}
\mathrm{P}_{q}^{*} & =\sum_{w \in \operatorname{Sym}(n)} \varphi_{q}(w)^{*} \sum_{t \in T} \varphi_{q}(t)^{*}=\sum_{w \in \operatorname{Sym}(n)} \varphi_{q}\left(w^{-1}\right)\left(\prod_{j \in[n]} \sum_{i \in[0, m-1]} \varphi_{q}\left(t_{j}\right)^{i}\right)^{*} \\
& =\sum_{w \in \operatorname{Sym}(n)} \varphi_{q}(w)\left(\prod_{j \in[n]} \sum_{i \in[0, m-1]} \varphi_{q}\left(t_{j}\right)^{i}\right)=\sum_{w \in \operatorname{Sym}(n)} \varphi_{q}(w) \sum_{t \in T} \varphi_{q}(t)=\mathrm{P}_{q} .
\end{aligned}
$$

Then, the map $q \mapsto \mathrm{m}_{0}\left(\mathrm{P}_{q}\right)$ is continuous, since the map $q \mapsto \mathrm{P}_{q}$ is norm-continuous and $\left|\mathrm{m}_{0}\left(\mathrm{P}_{q_{1}}\right)-\mathrm{m}_{0}\left(\mathrm{P}_{q_{2}}\right)\right| \leq\left\|\mathrm{P}_{q_{1}}-\mathrm{P}_{q_{2}}\right\|_{\text {op }}$. Remark that $\mathrm{P}_{0}=\mathrm{id}_{\mathbf{H}}$ and $\mathrm{P}_{1}=\mathrm{P}$. The invertibility of $P_{q}$ deduced from Lemma 1 implies $m_{0}\left(P_{q}\right) \neq 0$. As $m_{0}\left(P_{0}\right)=1$, we necessarily have $\mathrm{m}_{0}\left(\mathrm{P}_{q}\right)>0$, in particular $\mathrm{m}_{0}\left(\mathrm{P}_{1}\right)>0$.

Proposition 1. Let $\Pi=\left\{t_{1}, t_{2}, \ldots, t_{n}, s_{1}, s_{2}, \ldots, s_{n-1}\right\}$ be a representative reflection set of the complex reflection group $G(m, 1, n)$, and $\varphi$ the quasimultiplicative map of Theorem 1 . If $\|\varphi(r)\|_{\text {op }} \leq 1$ for every $r \in \Pi$, then the operator $\mathrm{P}=\sum_{u \in G(m, 1, n)} \varphi(u)$ is positive.

Proof. The argument is similar to that of [2, Theorem 2.2]. Let $\varphi_{q}: \mathbb{C} G(m, 1, n) \rightarrow \mathscr{B}_{\mathbf{H}}$, for $0 \leq q<1$, be the quasimultiplicative linear map in the proof of Lemma 2. We know from Lemma 2 that $\mathrm{P}_{q}:=\sum_{u \in G(m, 1, n)} \varphi_{q}(u) \in \mathscr{B}_{\mathbf{H}}$ is strictly positive. As $\lim _{q \rightarrow 1^{-}} \mathrm{P}_{q}=\mathrm{P}$ uniformly, we get the result.

For $v \in G(m, 1, n)$, let $\theta_{v}: \mathbb{C} G(m, 1, n) \rightarrow \mathbb{C} G(m, 1, n)$ be the operator

$$
\theta_{v}(f):=v f=\sum_{u \in G(m, 1, n)} f\left(v^{-1} u\right) u \quad \text { with norm } \quad\left\|\theta_{v}\right\|_{\text {op }}=1 .
$$

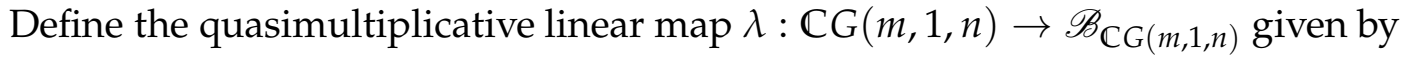

$$
\lambda(1)=\operatorname{id}_{\mathbb{C} G(m, 1, n)}, \quad \forall i \in[n-1]: \lambda\left(s_{i}\right)=\theta_{s_{i}} \quad \text { and } \quad \forall j \in[n]: \lambda\left(t_{j}\right)=\theta_{t_{j}} .
$$

One can easily verify that $\lambda\left(s_{i}\right)$ and $\sum_{i \in[m-1]} \lambda\left(t_{j}\right)^{i}$ are self-adjoint. We can now establish the proof of Theorem 1. 
Proof. For $i \in[n-1]$ and $j \in[n]$, define the operators $\hat{S}_{i}$ and $\hat{\mathrm{T}}_{j}$ on $\mathbb{C} G(m, 1, n) \otimes \mathbf{H}$ respectively by $\hat{S}_{i}:=\lambda\left(s_{i}\right) \otimes S_{i}$ and $\hat{T}_{j}:=\lambda\left(t_{j}\right) \otimes \mathrm{T}_{j}$. And let $\hat{\varphi}: \mathbb{C} G(m, 1, n) \rightarrow \mathscr{B}_{\mathbb{C} G(m, 1, n) \otimes \mathbf{H}}$ be the quasimultiplicative linear map given by

$\hat{\varphi}(1)=\mathrm{id}_{\mathrm{C} G(m, 1, n) \otimes \mathbf{H}^{\prime}} \quad \forall i \in[n-1]: \hat{\varphi}\left(s_{i}\right)=\lambda\left(s_{i}\right) \otimes \mathrm{S}_{i} \quad$ and $\quad \forall j \in[n]: \hat{\varphi}\left(t_{j}\right)=\lambda\left(t_{j}\right) \otimes \mathrm{T}_{j}$.

As $\left\|\hat{\varphi}\left(s_{i}\right)\right\|_{\text {op }} \leq 1$ and $\left\|\hat{\varphi}\left(t_{j}\right)\right\|_{\text {op }} \leq 1$, we deduce from Proposition 1 that the operator $\hat{\mathrm{P}}=\sum_{u \in G(m, 1, n)} \hat{\varphi}(u)$ is positive. Let $x_{1}, \ldots, x_{k} \in \mathbf{H}, f_{1}, \ldots, f_{k} \in \mathbb{C} G(m, 1, n)$, and set

$$
y=\sum_{u \in G(m, 1, n)} u \otimes \sum_{i \in[k]} f_{i}\left(u^{-1}\right) x_{i} \in \mathbb{C} G(m, 1, n) \otimes \mathbf{H} .
$$

Then, on the Hilbert space $\mathbb{C} G(m, 1, n) \otimes \mathbf{H}$, we have

$$
\begin{aligned}
0 \leq\langle\hat{\mathrm{P}} y, y\rangle & =\sum_{u \in G(m, 1, n)}\langle(\lambda(u) \otimes \varphi(u)) y, y\rangle \\
& =\sum_{u, v, w \in G(m, 1, n)} \sum_{i, j \in[k]}\left\langle(\lambda(u) \otimes \varphi(u))\left(v \otimes f_{i}\left(v^{-1}\right) x_{i}\right), w \otimes f_{j}\left(w^{-1}\right) x_{j}\right\rangle \\
& =\sum_{u, v, w \in G(m, 1, n)} \sum_{i, j \in[k]}\left\langle\theta_{u}(v) \otimes f_{i}\left(v^{-1}\right) \varphi(u) x_{i}, w \otimes f_{j}\left(w^{-1}\right) x_{j}\right\rangle \\
& =\sum_{u, v, w \in G(m, 1, n)} \sum_{i, j \in[k]}\langle u v, w\rangle\left\langle f_{i}\left(v^{-1}\right) \varphi(u) x_{i}, f_{j}\left(w^{-1}\right) x_{j}\right\rangle \\
& =\sum_{u, v, w \in G(m, 1, n)} \sum_{i, j \in[k]}\langle u v, w\rangle\left\langle\overline{f_{j}\left(w^{-1}\right)} f_{i}\left(v^{-1}\right) \varphi(u) x_{i}, x_{j}\right\rangle \\
& =\sum_{u, v \in G(m, 1, n)} \sum_{i, j \in[k]}\left\langle\overline{f_{j}\left(v^{-1} u^{-1}\right)} f_{i}\left(v^{-1}\right) \varphi(u) x_{i}, x_{j}\right\rangle \\
& =\sum_{i, j \in[k]}\left\langle\varphi\left(\sum_{u, v \in G(m, 1, n)} \frac{f_{j}\left(v^{-1}\right)}{\langle} f_{i}\left(v^{-1} u\right) u\right) x_{i}, x_{j}\right\rangle=\sum_{i, j \in[k]}\left\langle\varphi\left(f_{j}^{*} f_{i}\right) x_{i}, x_{j}\right\rangle .
\end{aligned}
$$

\section{Representation on Fock Space}

In this section, we prove that the sesquilinear form $\langle\cdot, \cdot\rangle_{G}$ in Theorem 2 is an inner product, for which suitable creation and annihilation operators are defined.

Recall the canonical free creation and annihilation operators $I^{*}(x): \bigoplus_{n \in \mathbb{Z}_{+}} \mathbf{H}^{\otimes n} \rightarrow \bigoplus_{n \in \mathbb{Z}_{+}} \mathbf{H}^{\otimes n}$ and I $(x): \bigoplus_{n \in \mathbb{Z}_{+}} \mathbf{H}^{\otimes n} \rightarrow \bigoplus_{n \in \mathbb{Z}_{+}} \mathbf{H}^{\otimes n}$ respectively, for each $x \in \mathbf{H}$, defined by [4, § 2]

$$
\begin{array}{lll}
\mathrm{I}^{*}(x) \Omega=x & \text { and } \quad \mathrm{I}^{*}(x) x_{1} \otimes \cdots \otimes x_{n}=x \otimes x_{1} \otimes \cdots \otimes x_{n} \\
\mathrm{I}(x) \Omega=0 & \text { and } \quad \mathrm{I}(x) x_{1} \otimes \cdots \otimes x_{n}=\left\langle x, x_{1}\right\rangle x_{2} \otimes \cdots \otimes x_{n}
\end{array}
$$

for $x_{1}, \ldots, x_{n} \in \mathbf{H}$. Consider the operator

$$
\mathrm{R}_{n}:=\left(\mathrm{id}_{\mathbf{H}^{\otimes n}}+\sum_{i \in[m-1]} \mathrm{T}_{1}^{i}\right)\left(\mathrm{id}_{\mathbf{H}^{\otimes n}}+\sum_{j \in[n-1]} \prod_{i \in[j]}^{\overrightarrow{ }} \mathrm{S}_{i}\right) \in \mathscr{B}_{\mathbf{H}^{\otimes n}} .
$$


We define the creation and annihilation operators $\mathrm{d}^{*}(x)$ and $\mathrm{d}(x)$ in Theorem 2 by

$$
\mathrm{d}^{*}(x):=\mathrm{I}^{*}(x) \quad \text { and } \quad \mathrm{d}(x) x_{1} \otimes \cdots \otimes x_{n}:=\mathrm{I}(x) \mathrm{R}_{n} x_{1} \otimes \cdots \otimes x_{n} .
$$

Remark that they are clearly not adjoint with respect to the usual inner product $\langle\cdot, \cdot\rangle$. We can now establish the proof of Theorem 2.

Proof. As stated in Lemma 2, $\mathrm{P}_{n}$ is strictly positive, then $\langle., \cdot\rangle_{G}$ is an inner product. By definition of $S_{i}$ and $\mathrm{T}_{j}$, we have $\mathrm{I}^{*}(x) \mathrm{S}_{i}=\mathrm{S}_{i+1} \mathrm{I}^{*}(x)$ and $\mathrm{I}^{*}(x) \mathrm{T}_{j}=\mathrm{T}_{j+1} \mathrm{I}^{*}(x)$, which implies

$$
I^{*}(x) \mathrm{P}_{n}=\left(\operatorname{id}_{\mathbf{H}} \otimes \mathrm{P}_{n}\right) I^{*}(x) \text { or } \mathrm{P}_{n} \mathrm{l}(x)=\mathrm{I}(x)\left(\mathrm{id}_{\mathbf{H}} \otimes \mathrm{P}_{n}\right) .
$$

Note that

$$
\mathrm{P}_{n+1}=\left(\sum_{u \in G(m, 1, n)} \varphi(u)\right)\left(\varphi(1)+\sum_{i \in[m]} \varphi\left(t_{1}^{i}\right)\right)\left(\varphi(1)+\sum_{j \in[n]} \prod_{i \in[j]}^{\rightarrow} \varphi\left(s_{i}\right)\right),
$$

where the complex reflection group $G(m, 1, n)$ is generated by $\left\{t_{2}, t_{3}, \ldots, t_{n+1}, s_{2}, s_{3}, \ldots, s_{n}\right\}$ as reflection set, $\sum_{u \in G(m, 1, n)} \varphi(u)=\mathrm{P}_{n}$, and $\left(\varphi(1)+\sum_{i \in[m]} \varphi\left(t_{1}^{i}\right)\right)\left(\varphi(1)+\sum_{j \in[n]} \prod_{i \in[j]}^{\overrightarrow{1}} \varphi\left(s_{i}\right)\right)=\mathrm{R}_{n+1}$. Then,

$$
\mathrm{P}_{n+1}=\left(\mathrm{id}_{\mathbf{H}} \otimes \mathrm{P}_{n}\right) \mathrm{R}_{n+1} .
$$

Now, for $X \in \mathbf{H}^{\otimes n}$ and $Y \in \mathbf{H}^{\otimes n+1}$, we have

$$
\begin{aligned}
\left\langle\mathrm{d}^{*}(x) X, Y\right\rangle_{G}=\left\langle\mathrm{d}^{*}(x) X, \mathrm{P}_{n+1} Y\right\rangle & =\left\langle X, \mathrm{I}(x) \mathrm{P}_{n+1} Y\right\rangle=\left\langle X, \mathrm{I}(x)\left(\operatorname{id}_{\mathbf{H}} \otimes \mathrm{P}_{n}\right) \mathrm{R}_{n+1} Y\right\rangle \\
& =\left\langle X, \mathrm{P}_{n} \mathrm{I}(x) \mathrm{R}_{n+1} Y\right\rangle=\left\langle X, \mathrm{P}_{n} \mathrm{~d}(x) Y\right\rangle=\langle X, \mathrm{~d}(x) Y\rangle_{G} .
\end{aligned}
$$

Moreover, since

$$
\left\|\mathrm{R}_{n+1}\right\|_{\mathrm{op}} \leq\left(\sum_{i \in[0, n]}\|\mathrm{S}\|_{\mathrm{op}}^{i}\right)\left(\sum_{j \in[0, m-1]}\|\mathrm{T}\|_{\mathrm{op}}^{j}\right) \leq \frac{1}{\left(1-\|\mathrm{S}\|_{\mathrm{op}}\right)\left(1-\|\mathrm{T}\|_{\mathrm{op}}\right)},
$$

then

$$
\left\|\mathrm{P}_{n+1} \mathrm{P}_{n+1}\right\|_{\text {op }}=\left\|\left(\mathrm{id}_{\mathbf{H}} \otimes \mathrm{P}_{n}\right) \mathrm{R}_{n+1} \mathrm{R}_{n+1}^{*}\left(\mathrm{id}_{\mathbf{H}} \otimes \mathrm{P}_{n}\right)\right\|_{\mathrm{op}} \leq \frac{\left\|\left(\mathrm{id}_{\mathbf{H}} \otimes \mathrm{P}_{n}\right)\left(\mathrm{id}_{\mathbf{H}} \otimes \mathrm{P}_{n}\right)\right\|_{\text {op }}}{\left(1-\|\mathrm{S}\|_{\mathrm{op}}\right)^{2}\left(1-\|\mathrm{T}\|_{\mathrm{op}}\right)^{2}}
$$

hence $\left\|\mathrm{P}_{n+1}\right\|_{\mathrm{op}} \leq \frac{\left\|\left(\mathrm{id}_{\mathbf{H}} \otimes \mathrm{P}_{n}\right)\right\|_{\mathrm{op}}}{\left(1-\|\mathrm{S}\|_{\mathrm{op}}\right)\left(1-\|\mathrm{T}\|_{\mathrm{op}}\right)}$. Denoting by $\|\cdot\|_{G}$ the norm associated to $\langle\cdot, \cdot \cdot\rangle_{G}$, for $X \in \mathbf{H}^{\otimes n}$, we consequently have

$$
\begin{aligned}
\left\|\mathrm{d}^{*}(x) X\right\|_{G} & =\left\langle\mathrm{d}^{*}(x) X, \mathrm{~d}^{*}(x) X\right\rangle_{G}=\langle x \otimes X, x \otimes X\rangle_{G}=\left\langle x \otimes X, \mathrm{P}_{n+1} x \otimes X\right\rangle \\
& \leq \frac{1}{\left(1-\|\mathrm{S}\|_{\mathrm{op}}\right)\left(1-\|\mathrm{T}\|_{\mathrm{op}}\right)}\left\langle x \otimes X,\left(\mathrm{id}_{\mathbf{H}} \otimes \mathrm{P}_{n}\right) x \otimes X\right\rangle \\
& =\frac{1}{\left(1-\|\mathrm{S}\|_{\mathrm{op}}\right)\left(1-\|\mathrm{T}\|_{\mathrm{op}}\right)}\langle x, x\rangle\left\langle X, \mathrm{P}_{n} X\right\rangle_{G}=\frac{\|x\|\|X\|_{G}}{\left(1-\|\mathrm{S}\|_{\mathrm{op}}\right)\left(1-\|\mathrm{T}\|_{\mathrm{op}}\right)} .
\end{aligned}
$$




\section{References}

[1] Bargmann V. Remarks on a Hilbert Space of Analytic Functions. Proc. Nat. Acad. Sci. USA 1962, 48 (2), 199-204. doi:10.1073/pnas.48.2.199

[2] Bożejko M., Speicher R. Completely Positive Maps on Coxeter Groups, Deformed Commutation Relations, and Operator Spaces. Math. Ann. 1994, 300, 97-120. doi:10.1007/BF01450478

[3] Daletskii A., Kalyuzhny A., Lytvynov E., Proskurin D. Fock Representations of Multicomponent (particularly non-Abelian Anyon) Commutation Relations. Rev. Math. Phys. 2020, 32 (5), 2030004. doi:10.1142/S0129055X20300046

[4] Evans D. On On. Publ. Res. Inst. Math. Sci. 1980, 16 (3), 915-927. doi:10.2977/PRIMS/1195186936

[5] Greenberg O. Example of Infinite Statistics. Phys. Rev. Lett. 1990, 64 (7), 705-708. doi:10.1103/PhysRevLett.64.705

[6] Kassel C. Quantum Groups. In: Vakil R. (Ed.) Graduate Texts in Mathematics, 155, New York, 1995.

[7] Mathas A., Orellana R. Cyclotomic Solomon Algebras. Adv. Math. 2008, 219 (2), 450-487.

[8] Meljanac S., Svrtan D. Study of Gram Matrices in Fock Representation of Multiparametric Canonical Commutation Relations, extended Zagier's Conjecture, Hyperplane Arrangements and Quantum Groups. Math. Commun. 1996, 1 (1), 1-24.

[9] Randriamaro H. A Multiparametric Quon Algebra. Bull. Iranian Math. Soc. 2021, 47 (3), 843-854. doi: $10.1007 /$ s41980-020-00414-5

[10] Speicher R. Generalized Statistics of Macroscopic Fields. Lett. Math. Phys. 1993, 27, 97-104.

[11] Stochel J. Representation of Generalised Creation and Annihilation Operators in Fock Space. Univ. Iagel. Acta Math. 1997, 34, 135-148.

[12] Zagier D. Realizability of a Model in Infinite Statistics. Comm. Math. Phys. 1992, 147, 199-210. doi:10.1007/BF02099535

Received 06.10.2020

Revised 10.08.2021

Рандріамаро Г. Цілком позитивні відображення для непримітивних комплексних груп відбиття // Карпатські матем. публ. — 2021. - Т.13, №2. - С. 452-459.

М. Божейко та Р. Шпайхер у 1994 році довели існування цілком позитивних квазімультиплікативних відображень з групової алгебри груп Коксетера у множину обмежених операторів. Вони використали деякі з них для того, щоб визначити скалярний добуток, асоційований з операторами народження та знищення, заданими на прямій сумі тензорних степенів гільбертового простору, що має назву повного простору Фока. Згодом у 2008 році А. Матхас та Р. Ореллана визначии функцію довжини на непримітивних комплексних групах відбиття, що дозволило їм ввести аналог алгебри спуску груп Коксетера. У цій статті ми використовуємо функцію довжини для того, щоб розширити результат М. Божейка та Р. Шпайхера на непримітивні комплексні групи відбиття. Іншими словами, ми доводимо існування цілком позитивних квазімультиплікативних відображень з групової алгебри непримітивних комплексних груп відбиття у множину обмежених операторів. Аеякі з цих відображень пізніше використовуються для визначення більш загального скалярного добутку, що асоційований з операторами народження та знищення на повному просторі Фока. Нагадаємо, що у квантовій механіці стан фізичної системи представляється вектором гільбертового простору, а оператори народження та знищення діють на фоківський стан як відповідно додавання та відкидання частинки у приписаному квантовому стані.

Ключові слова і фрази: обмежений оператор, квазімультиплікативне відображення, простір Фока. 